\title{
LEARNING IN PUBLIC AND A PATH TOWARDS METHODOLOGICAL ACTIVISM: A CONVERSATION ON EQUITY RESEARCH
}

\author{
Julie P. Martin, ${ }^{1, *}$ Shannon K. Stefl, ${ }^{2}$ E Amy E. Slaton ${ }^{3}$ \\ ${ }^{1}$ Department of Engineering Education, The Ohio State University, Columbus, \\ Ohio 43210, USA \\ ${ }^{2}$ Office of Teaching Effectiveness and Innovation, Clemson University, Clemson, \\ South Carolina 29634, USA \\ ${ }^{3}$ Department of History, Drexel University, Philadelphia, Pennsylvania 19104, \\ USA
}

*Address all correspondence to: Julie P. Martin, Department of Engineering Education, The Ohio State University, Columbus, OH 43210; Tel.: +614-292-7923, E-mail: martin.4071@osu.edu

\begin{abstract}
In this position paper, we propose ways that the engineering education research community can engage in a form of methodological activism whereby researchers intentionally choose methods for the political purpose of empowering marginalized populations. We discuss the evolution of our thinking on these topics in a manner which we call "learning in public." We start this narrative with the federally funded working conference that brought us together as a research team and offer a call to action through discussion of theory, methods, and research questions that encompass methodological activism. We acknowledge that many disincentives exist to making these changes, and we offer fellow researchers encouragement and support in the form of a formal research citation; the provision of substantive ideas for critical framings in association with a peer-reviewed publication is significant. We assert that adoption of these research practices, facilitated through the conscious deployment of scholarly credibility, will push the field towards a more justice-oriented research practice. We invite other researchers to join us in this aim, as we join those already conducting such work. We hope this publication will spark continued debate and evolution will occur through future discussions and critiques.
\end{abstract}

KEY WORDS: methodological activism, engineering education, justice-oriented research

\section{INTRODUCTION}

We consider current ideas about what can and cannot be legitimately asked in the context of academic research in the United States on diversity, equity, and inclusion (DEI) in engineering education in this position paper. We talk about whose identity remains legitimized in engineering (i.e., people seen to be male, White, heterosexual, abled, and native-born Americans) and who continues to be seen as illegitimate (Cross, 2020). Interrogating such active formulations of difference is necessary to any projects of reform, or reparation, in spaces where inequity has historically brought about marginality or harm (we note that it is not sufficient to those goals). This paper represents, in fact, an act of attempted legitimation itself. In the following pages we acknowledge and attempt 
to exploit our positions as credentialed, funded scholars, and as people who identify as White, cis, and abled in order to support critical interrogations of currently delegitimized and marginalized researchers and research topics. These topics include those conditions that render some people - commonly people possessing majority background, such as those of we authors - and not other people as likely recipients of research resources and associated rewards. The experiences of marginalized people in engineering settings include, but are not limited to, encounters with anti-Black conducts, homophobia, classism, xenophobia, and ableism, but academia tends to dismiss such experiences in favor of more ameliorative renderings of "stereotype bias," "chilly climates," or even more perniciously, "low student efficacy." Such labels present more palatable alternatives to confronting the brutal experiences that marginalized students, staff, and faculty report, framing those experiences as regrettable and anomalous rather than as problems endemic to engineering education.

In conventional DEI research, the sorting of engineering aspirants into familiar categories (e.g., by race, gender, sexuality, disability, socioeconomic status) for the purposes of data collection and analysis itself reproduces the privileged status of many researchers (Berrey, 2015; Ahmed, 2012). We are troubled by the taxonomies long-established by research and funding institutions. We realize that labels facilitate critical inquiry around equity in some instances. In other instances, however, methods of sorting and labeling obscures complex, intersectional self-identifications and leaves majority communities and their historical authority unproblematized.

With these epistemic disruptions in mind and with an end goal of a more just educational system with more equitable labor practices, we assert that the established institutional metrics of rigorous, valuable engineering-equity research must be challenged. We write in support of "methodological activism" (Ong, 2005; Zuberi and Bonilla-Silva, 2008) by which research methods purposefully empower marginalized populations and enact social change. The working conference on which this paper is based, and the following text, came into being through the collective efforts of many people beyond the three named authors. Some of these additional researchers have been named in the roster of conference participants and subsequent "critical friends" who provided feedback and guidance. Numerous others make up the communities in which the we have learned, grown, and worked; the conventions of academic authorship and its limits are in no sense beyond the concerns of this essay.

\subsection{Positionality of the Authors}

The convention of positionality statements leads us to indicate that we, the authors, identify as White, abled, cisgender women, securely employed within institutions of higher education. We wish to expand the content and conventions of positionality statements, however, to reflect on the process of inquiry and writing as it may reproduce privilege. We contend that readers should never accept a reporting of research without critical reflection on the process through which the findings emerged. Such divisions between findings and processes reassert power and marginalization. Researchers need 
to take steps beyond making explicit their institutional locations, methods, and aims to be truthful. The disclosure of research positionality should confront the messiness of research centered on the human experience. Clean, neatly bounded findings reflect neither the social realities we study nor the facilities and difficulties that different individuals face in undertaking research, reflection, and revision.

We also believe that, if we are to solve problems of inequity, we researchers must recognize the many complex ways in which human difference has shaped engineering learning and work in the United States, even though dominant cultural narratives have described these settings as free of social and ideological commitments based on identity. We do not believe that identities are stable or singular in our culture. This belief is captured by aspects of intersectionality (Crenshaw, 1989), highlighting the powerful role of time and place in all experiences of difference. Intersectional analysis reminds us that our understanding of selves and others as having a race, gender, sexuality, or as abled or disabled minds or bodies, derives from social conditions, not from unchanging innate characteristics of individuals (Grzanka, 2014). We have sought to create a foundation for critical conversations on diversity research by articulating the nature of difference in U.S. engineering, as well as articulating particular experiences of particular differences.

Throughout the initial working conference on which we reflect and throughout writing this paper, our disposition has been one of iterative self-reflection and sharing. This "learning-in-public" stance of uncertainty required us to transgress familiar structures of privilege and remain open, at all junctures, to input and critique. We use these pages to critically reflect on, and indeed problematize, the process by which our research findings took shape. We offer broader statements about the directions in which future research may progress, and we acknowledge the structures that continue to limit the possibilities of such work.

\subsection{Origins}

We began developing the areas of focus and calls to action on which this project was based in a National Science Foundation (NSF)-sponsored working conference entitled "Who's Not at the Table? Building Research Capacity for Underserved Communities in Engineering," held in 2016 at Clemson University. Seventy scholars, policymakers, and practitioners from diverse fields and backgrounds came together for a two-day working conference with the goal of framing new possibilities for "broadening participation" research in engineering education; with this term, the NFS tends to refer to research on the correction of the numerical underrepresentation of communities.

Our learning in public began more than a year before the actual conference event. This beginning is important because the origin point illuminates whose experiences were even considered observable from the perspective of federal funding agencies. Starting here demonstrates the narrowed social/conceptual boundaries within which researchers operate. Julie and Amy were invited to the NSF headquarters to develop a proposal that would contribute new dimensions to national conversations about broadening participation research. At that time the NSF already had a portfolio of projects of this kind 
focused on race and gender, so we were instructed to bring other, understudied identities into critical focus, namely, disabled people, veterans, low-income/first-generation college goers (LIFGd), and persons of lesbian, gay, bisexual, transgender, intersex, or queer (LGBTQ+) identity.

The NSF's project portfolio looked quite different in 2016 than it does now in 2021. In 2016, the Engineering Directorate had not placed as much focus on people with disabilities as it has today. Limited funding investments had been made for the study of first-generation college students (e.g., Lucena et al., NSF-1354087; Martin, NSF0950710) and members of the LGBTQ+ community (e.g., Farrell et al., NSF-1539140). Investments in broadening participation by veteran populations mostly supported education and career development for individual veterans (NSF, 2014), but research to explore systemically broadening their participation in engineering had been awarded almost no funding (e.g., Lord et al., NSF-1428512 and 1428646). Although the identities on which we were asked to focus were widely understood to be marginalized in engineering, these identities had not been officially recognized by the federal definition of "underrepresented."

We knew that attempts to address each identity-(dis)ability, LGBTQ+, veteran, and LIFG-individually would be deeply theoretically flawed, so we chose to frame discussions throughout the working conference from an intersectional perspective, emphasizing arrays of identities as they related to engineering education research. We intentionally recruited conference participants who self-identified with a range of racial/ ethnic, gender, sexual, ability, and professional embodiments. In doing so we sought perspectives of people who embodied the identities we aimed to discuss, knowing that their perspectives were complimented with the perspective of others who did not embody these identities. The resulting combined, complex reflections expanded past the artificial identity boundaries the funding agency had put in place. However, in staging the event, we adhered to NSF directives to expand the notion of underrepresentation of primarily the specified identities; we did not directly engage in reflection on race and gender (though we also did not discourage that reflection). We now realize that we would have supported a fuller and more robust exchange had we explicitly invited race and gender framings into the conversation. We see this as our main learning-in-public takeaway from organizing the working conference.

Our thinking evolved from staging and reporting on the event itself to writing this position paper. As an author group, we gained a deeper understanding of how mandated definitions of underrepresentation used by funding agencies, themselves constrained by congressional oversight, can (re)produce privilege by determining what social identities warrant attention from the research community. As social science researchers, we were familiar with the desire of funding agencies for samples crystallized by well-defined boundaries between social identities and among associated educational experiences; we are acutely aware that these tidy realities do not exist (Slaton and Pawley, 2018). We were excited to bring inquiry into severely understudied areas, but we also felt increasingly uncomfortable with how agencies, deeply politicized due to congressional oversight, constrain investigations. Our responsibility now is to join fellow scholars who 
have sought to problematize the boundaries among social identities (say, those on which "diversity" initiatives may readily be predicated) and mechanisms of marginalization (Haverkamp et al., 2021; Kliewer et al., 2015; Ladson-Billings, 1998). Thus, our reporting necessarily must expand beyond the organizing precepts of the NSF-funded event. Next, we share what we learned with participants regarding theoretical, methodological, and research question considerations in DEI research and how this piece can enact that expansion.

\section{ENVISIONING THE FUTURE OF ENGINEERING EDUCATION RESEARCH IN THE WORKING CONFERENCE}

This position paper is the product of a multiyear process. We hope it stands as a starting point for sustained change in engineering higher education. Participants at the working conference reflected on ways in which engineering education research has perpetuated inequitable structures, developing questions about the changes and expansions in research settings and in communities of practice that could lead to proportionate participation in engineering across all groups. Discussions were focused around five threads: theories, research methods, research questions, educational practices and experience, and knowledge needed to inform practice. Participants worked together to synthesize ideas arising from discussion of the threads. They wrote, drew, talked, and shared through closely structured exercises and freewheeling debates around meeting tables and at fireside chats. Detailed descriptions of the working conference findings for each of these threads can be found in our other published materials (e.g., Martin et al., 2017, 2018). In these papers we outlined specific calls to action arising from the communitygenerated findings. We encourage researchers looking to align their projects with the future envisioned by the community to explore the detailed reports listed above.

After the conference, we invited scholars from inside and outside the field to serve as "critical friends" for the project by providing challenging analysis. Some of the critical friends had participated in the working conference while others were new to the project. The critical friends reflected in writing on a set of questions about the document draft; we then participated in a professionally facilitated "circle of trust" (Palmer, 2017). A Quaker tradition where everyone's voice is valued equally, the circle of trust is a process of shared exploration that brought us back to the integrity of our shared vision and community input. Following the circle of trust, we revisited the text of this paper to incorporate the critical feedback we had received. The final formative element of this document was the peer review process for publication in a journal (Walther et al., 2013).

As we expanded our thinking beyond immediate conference outcomes, we recognized that the metaphor that we used in the original conference title "who's not at the table" was itself likely to reproduce structures we hoped to reshape. The idea that existing institutions, epistemics, and practices (i.e., "the table") do not need reformation, and that the inclusion of previously absent individuals in these settings will enact desired social change, is one we do not accept. Similarly, we turned away from the term "underrepresentation," choosing to use "minoritized" and "marginalized" to indicate agency on 
Martin, Stefl, \& Slaton

the part of existing institutions and privileged actors therein. These terms in the literature are evolving, and we do not presume that they will remain optimal for future critical projects (Coley et al., 2021; Long III, 2020). This position paper remains true to the rich community discussions centered on the identities of specific focus during and after the working conference, yet we are compelled to expand past our charged task and acknowledge the broader social, political, and racial climate operating within the U.S. in 2021. The innumerable array of identities intersecting with those initially centered cannot be set aside; thus we offer a wider narrative relevant to praxis in today's social climate.

\subsection{Call to Action}

Ideally, we wanted this paper to support others' "learning in public" about equity and institutional conducts in 2021. That support must include the legitimation of research strategies, agendas, and findings that do not skirt the messiness of intervening in unjust sectoral conditions (such as inequitable funding and employment in research). That legitimation, we hope, will not only help to bring anti-Black conducts, homophobia, classism, xenophobia, and ableism new standing as engineering education research topics, but also help to enable reflection about how those dispositions currently limit the involvement of minoritized people in the work of research (Holly Jr., 2020).

\subsubsection{Area 1: Theoretical Considerations}

All theory is political. That is, the framings with which we approach our empirical work allow and disallow the recognition of various phenomena. Because theories are deeply rooted in social norms, the theories we employ can reproduce discrimination, often erasing experiences of participants who differ from what is considered normative. Using theories that incorporate historical perspectives can be a powerful tool to challenge underlying social structures. Engineering education researchers may need to engage in (interdisciplinary) research collaboration to understand and apply theoretical tenets appropriately (Teti and Slaton, 2020).

Theory influences the structure of a study as well as the interpretation of observed phenomena. Researchers should examine whether existing theory is appropriate and sufficient. For example, deficit models that forestall effective address of inequitable conditions (Harry and Klingner, 2007; Harper, 2010; Slaton, 2010; Haverkmap et al., 2021) are all too common (explicitly or implicitly) in the framing of research questions. Determining the appropriateness of theory includes interrogating it for traces of deficit thinking, as well as for the ways it might deny non-normative experiences. If a selected theory does not support the well-being of a community, or if it retraumatizes individuals or decenters a community's historical experience, researchers should consider not using it (Mejia et al., 2018).

We call for researchers to consider the role of theory in inclusive engineering education study, such as research that systematically

- Questions and explores the appropriateness of using theory. 
- Advocates for counter theories or critical theories.

- Explores and tests the inherent limitations of existing theories by clearly stating and clarifying its limitations.

- Uses theoretical paradigms that originate from non-White or non-Western contexts (i.e., pro-Black, decolonializing, feminist, queer, or crip theories that decenter the importance of historically influential actor groups).

- Accepts that there may be no existing theory of suitable character.

- Develops and applies new theory.

- Uses asset-based theories and decries deficit-based theoretical stances.

These approaches will help researchers recognize patterns across social conditions or political episodes, articulate unexpected causal factors in observed phenomena, and reflect on their own theory selection and evaluation of evidence, especially as these actions are shaped by a researcher's assumptions about study participants.

\subsubsection{Area 2: Methodological Considerations}

Like theory, research methods are invariably political. Methods determine who is visible and invisible in the data, whose voice is heard and whose voice is silenced, and what changes in practice or policy should occur as a result of the research. Left unscrutinized, methods and methodologies can (re)produce power, privilege, and oppression. We call for engineering education researchers to examine their chosen methods/methodology, make use of empowering methodologies already in regular use outside of engineering, and to develop new ones as needed. This examination will require deep reflection (Litzler et al., 2019). We imagine an entire community of researchers engaged in methodological activism (Ong, 2005; Zuberi and Bonilla-Silva, 2008) where research methods are purposefully used to empower marginalized populations and enact social change. Doing this will require a reframing of research ethics (Sochacka et al., 2018), including developing new, relational approaches that authentically engage participants (Patrick et al., 2022, in press; Haverkamp et al., 2021; Martin and Garza, 2020; Secules et al., 2018; Smith and Lucena, 2016), as well as finding unconventional ways to present findings that are appropriate to honor participants' lived experiences (Morton and Parsons, 2018).

We also recognize that researchers must be funded in equitable ways to "democratize the research process" (Holly Jr., 2020, p. 631). We envision engineering education research proposals and publications in which researchers interrogate and publicly reveal their position in relation to systems of power, privilege, and oppression and consider how "choices in research topic, methodology, and communication" are shaped by their perspective (Secules et al., 2021, p. 38).

We call for the engineering education community to undertake research that

- Includes a critical examination of the underlying values, social and historical contexts, and assumptions of a chosen method/methodology.

- Intentionally uses methods/methodologies as a form of activism to achieve a political purpose.

Volume 28, Issue 1, 2022 
- Develops new methods/methodologies with the potential to empower research participants and their communities.

- Includes authentic, equitable involvement from persons embodying marginalized identities.

- Recognizes marginalized persons as experts in their own experiences.

- Includes deep reflection of the researcher's position in relation to systems of power, privilege, and oppression and its influence on the work.

These approaches will move the research community towards conceptual, evidentiary, and other sorts of methodological choices predicated on a widened notion of investigative rigor, one which incorporates the aims of equitable and justice-oriented practice as outlined above.

\subsubsection{Area 3: Research Questions to Ask}

We soundly reject research questions based on deficit thinking (Valencia and Solórzano, 1997). Such questions, which presume individuals from marginalized groups require reformulation or skill development for full institutional inclusion, shield the systems and have been set up to benefit individuals who are insiders of dominant culture. We call for antideficit research questions (Harper, 2010; Battey and Leyva, 2016) that focus on assets of marginalized participants, critique the system and culture, or both. We believe that research questions should break down normative culture on university campuses and in other engineering environments.

We call for the engineering education community to utilize research questions that

- Ask questions that avoid deficit framing of marginalized individuals and groups.

- Critique the system within which participants and the study are situated and not the individual participant, their family, or community.

- Name and explore conditions such as anti-Blackness, homophobia, and ablism that are customarily cast as "beyond" the purview of equity research.

- Avoid appropriating the experiences and priorities of minoritized people in the framing of research projects or desired outcomes.

As with the expanded and reflective approach to choices of theory or methodology suggested above, this approach to the formulation of research questions will support a justice-oriented research practice.

\section{WHERE WE GO FROM HERE}

There are many disincentives to making these changes. With this publication we join other scholars, many of whom are marginalized, who have been leading this work for a long time. Their work, at the very least, should be amplified by humbly offering a citation - the currency of academia - to partially legitimize a new evolution of engineering education research. By combining our own training and credentials, and the existing 
platform provided by this journal, we seek to help counter some of the "politics of respectability" that disallows challenge and discomfort. Those politics have historically cast conduct and ideas that are disruptive of White supremacy or domination as illegitimate or fanciful (Singh, 2004). The feedback system whereby reviewers help create criteria for funding, publication, and the award of other opportunities encourages us to address the understanding that reviewers have of their own influence, their own contribution to the politics of respectability (Coley et al., 2021). Potentially, this becomes a transformative activity, confronting marginalizing aspects of our research ecosystem. Similarly, because experts serve not just as researchers and reviewers but as educators of future experts, this project may have still wider pedagogical impacts.

We view this systematic effort at reflection as a beginning of a movement towards methodological activism, not an end to a project. In today's political climate, the acknowledgment of systemic marginalization in education continues to be under attack; renewed false narratives of meritocracy are still the loudest in the culture of higher education and engineering (Riley, 2017). Despite rhetoric of academic "racial reckoning" since 2020, the extraordinary acceleration of programs of austerity in higher education has not only led to unprecedented faculty and student precarity - disproportionately affecting members of minoritized communities - but has further undermined the redirection of resources towards projects of equity. Improving the culture within engineering education and research is the responsibility of all, not solely of those who shoulder the burden of exclusion. As those who have enjoyed great benefit from the existing structures, we are also obligated to use the power inequitably granted to us to disclose these structures and professional norms. Now, more than ever, we researchers and academics must leverage our positions of privilege and influence to combat exclusionary narratives within higher education. If we are all willing to do this, then visibility and incorporation of all (future) engineers' perspectives into the ecosystem and accompanying narratives of engineering may no longer remain a secondary or tertiary consideration.

As a research community, we believe we can embed the recommended principles of methodological activism into engineering education and research. Such an evolution would

- Challenge the overt and covert diminishment of minoritized groups' presence within engineering as well as the silencing of these individuals' voices within educational research.

- Better equip future engineering professionals and educators to face the broader political, economic, and social contexts surrounding, and inescapably informing, the cultivation of today and tomorrow's engineers, thereby producing broader forms of engineering expertise.

- Create space for authentic partnership with presently underserved communities in engineering higher education.

- Improve standards of transparency and positionality in research publications and expand them to expect reflections on the research process.

- Articulate to younger and future generations of engineers (and those who work to study and understand them) an increased value of their diverse presence with the field.

Volume 28, Issue 1, 2022 
One more element of this evolution could be articulated. We see this paper as an initial move that will support its interventionist aims only if the field of engineering education research turns towards these plans and also gathers resources, restructuring social and institutional opportunities for others, going forward. Put differently, with this paper we outline our aims, but at the same time, in the field of engineering education research, the very question of who the "we" is in any project, who is in a position to make claims and take action, and above all, who may best plan a just and equitable form for the field, requires our critical attention. And so we issue this invitation: How might we take up this critical attention together?

\section{ACKNOWLEDGMENTS}

We gratefully acknowledge the original working conference participants and critical friends who were instrumental in our "learning in public," named below. We could not have done this without Dr. Joy A.J. Howard's writing coaching, editing, and facilitating the circle of trust. This material is based upon work supported by the National Science Foundation under Grant No. EEC-1551605. Any opinions, findings, and conclusions or recommendations expressed in this material are those of the author(s) and do not necessarily reflect the views of the National Science Foundation.

\section{CRITICAL FRIENDS}

Alice Pawley, Purdue University; Ayush Gupta, Homi Bhabha Center for Science Education; Claire Horner Devine, Counterspace Consulting; Donna Riley, Purdue University; James Holly Jr., Wayne State University; Kelly Cross, University of Nevada, Reno; Trina Fletcher, Florida International University; Walter Lee, Virginia Tech

\section{WORKSHOP PARTICIPANTS}

Names and institutions are listed according to conference registration records; some names or affiliations have changed since that time:

Abel Chávez, Western State Colorado University; Adam Kirn, University of Nevada, Reno; Alfredo Cruz, Polytechnic University of Puerto Rico; Alice Pawley, Purdue University; Ana Dison, The University of Texas at Austin; Anastasia Rynearson, Purdue University; Andrea Ogilvie, Virginia Tech; Angela Minichiello, Utah State University; Anne Pollock, Georgia Tech; Araceli Martinez Ortiz, Texas State University; Brooke Coley, Arizona State University; Bruce Wellman, Engineering Academy at Olathe Northwest High School; C. LaShan Simpson, Mississippi State University; Cara Lammey, University of Colorado Boulder; Cara Margherio, University of Washington; Catherine Mobley, Clemson University; Christopher Lim, Yale University; Cleo Woelfle-Erskine, UC Santa Cruz; Cynthia Hampton, Virginia Tech; Dan Standage, Student Veterans of America; Darryl Williams, Mississippi State University; David Silverstein, University of Kentucky; Deborah Grzybowski, The Ohio State Uni- 
versity; Donna Korbel, University of Connecticut; Donna Riley, Virginia Tech; Elizabeth Litzler, University of Washington; Emily Liptow, Cal Poly, San Luis Obispo; Fredericka Brown, The University of Texas at Tyler; Héctor Rodríguez-Simmonds, Purdue University; Holly Matusovich, Virginia Tech; Ian Smith, Independent; James Moore, National Science Foundation; Jane Dong, California State University-Los Angeles; Janice Hall, Virginia Tech; John Ristvey, UCAR Center for Science Education; Juan Lucena, Colorado School of Mines; Karl Booksh, University of Delaware; Karlyn Adams-Wiggins, The University of Texas at Tyler; Kelly Cross, University of Illinois Urbana-Champaign; Kristine Kozuch, Springfield Technical Community College; Leyva, Luis, Vanderbilt University; Lindi Rubadiri-Mujugira, Bellevue College; Lisa Massi, University of Central Florida; Liz Cox, Red Rocks Community College; Lyla Crawford, University of Washington; Mallory Chua, Olin College; Manu Platt, Georgia Institute of Technology; Maria del Pilar O'Cadiz, University of California-Los Angeles; Marlon Mitchell, University of Illinois Urbana-Champaign; Martina Svyantek, Virginia Tech; Mei-Fang Lan, University of Florida/CWC; Miguel Velez Reyes, The University of Texas at El Paso; Nadia Kellam, Arizona State University; Rocio Chavela Guerra, American Society for Engineering Education; Ryan Berke, Utah State University; Sarah Woodruff, Miami of Ohio; Shawn Jordan, Arizona State University; Shawna Fletcher, Texas A\&M University; Sheryl Burgstahler, University of Washington; Stephanie Farrell, Rowan University; Stephanie Zywicki, Purdue University; Stephen Secules, University of Maryland; Timothy Atherton, Tufts University; Trina Fletcher, National Society of Black Engineers; Wolfgang Sigmund, University of Florida; Yvette Pearson Weatherton, Rice University

\section{REFERENCES}

Ahmed, S. (2012). On being included: Racism and diversity in institutional life. Durham, NC: Duke University Press.

Berrey, E. (2015). The enigma of diversity: The language of race and the limits of racial justice. Chicago, IL: University of Chicago Press.

Battey, D., \& Leyva, L. A. (2016). A framework for understanding Whiteness in mathematics education. Journal of Urban Mathematics Education, 9(2), 49-80.

Coley, B. C., Simmons, D. R., \& Lord, S. M. (2021). Dissolving the margins: LEANING INto an antiracist review process. Journal of Engineering Education, 110(1), 8-14. DOI: 10.1002/jee.20375

Crenshaw, K. (1989). Demarginalizing the Intersection of Race and Sex: A Black Feminist Critique of Antidiscrimination Doctrine, Feminist Theory and Antiracist Politics. University of Chicago Legal Forum. 140, 139-67.

Cross, K. J. (2020). Racism is the manifestation of White supremacy and antiracism is the answer. Journal of Engineering Education, 109(4), 625-8. DOI: 10.1002/jee.20362

Farrell, S., Minerick, A., Cech, E., Chavela Guerra, R., \& Waidzunas, T. (2015). EAGER: Promoting LGBTQ Equality in Engineering through Virtual Communities of Practice, National Science Foundation Award No. 1539140.

Grzanka, P. (2014). Intersectionality: A foundations and frontiers reader. Boulder, CO: Westview Press.

Harper, S. R. (2010). An anti-deficit achievement framework for research on students of color in STEM. New Directions for Institutional Research, 2010(148), 63-74. DOI: 10.1002/ir.362

Harry, B., \& Klingner, J. (2007). Discarding the deficit model. Educational Leadership, 64(5), 16-21.

Volume 28, Issue 1, 2022 
Haverkamp, A., Bothwell, M., Montfort, D., \& Driskill, Q. L. (2021). Calling for a paradigm shift in the study of gender in engineering education. Studies in Engineering Education, 1(2), 55-70. DOI: 10.21061/see.34

Holly Jr., J. (2020). Disentangling engineering education research's anti-Blackness. Journal of Engineering Education, 109(4), 629-35.

Kliewer, C., Biklen, D., \& Petersen, A. (2015). At the end of intellectual disability. Harvard Educational Review, 85(1), 1-28. DOI: 10.17763/haer.85.1.j260u3gv2402v576

Ladson-Billings, G. (1998). Just what is critical race theory and what's it doing in a nice field like education? International Journal of Qualitative Studies in Education, 11(1), 7-24.

Litzer, E., Margherio, C., Affolter, E., \& Knaphus-Soran, E. (2019). Critical research questioning guide. Retrieved from http://depts.washington.edu/cerse/wordpress/wp-content/uploads/2019/06/CriticalResearch-Questioning-Guide_UW-CERSE_June-2019.pdf.

Long III, L. L. (2020). Toward an antiracist engineering classroom for 2020 and beyond: A starter kit. Journal of Engineering Education, 109(4), 636-9.

Martin, J. P., \& Garza, C. (2020). Centering the marginalized student's voice through autoethnography: Implications for engineering education research. Studies in Engineering Education, 1(1), 1-19. DOI: $10.21061 /$ see. 1

Martin, J. P., Stefl, S. K., \& Slaton, A. (2017). Developing a national research agenda: A data collection and community engagement model. In Proceedings of the 2017 American Society for Engineering Education Annual Conference and Exhibition.

Martin, J. P., Slaton, A., \& Stefl, S. K. (2018). Beyond inclusion, beyond welcome: Framing intersection research in engineering education. Retrieved from https://inclusiveengineering.files.wordpress. com/2021/07/framing_intersectional_research_in_engineering_education-2.pdf.

Mejia, J. A., Revelo, R. A., Villanueva, I., \& Mejia, J. (2018). Critical theoretical frameworks in engineering education: An anti-deficit and liberative approach. Education Sciences, 8(4), 158.

Morton, T. R., \& Parsons, E. C. (2018). \# BlackGirlMagic: The identity conceptualization of Black women in undergraduate STEM education. Science Education, 102(6), 1363-93.

National Science Foundation. (2014). Women, minorities, and persons with disabilities in science and engineering, NSF 14-124. National Science Foundation.

National Science Foundation. (2015). Dear colleague letter: Veterans Research Supplement (VRS) program, NSF 15-311. National Science Foundation.

Ong, M. (2005). Achieving multi-sitedness and social justice: "Methodological activism" and other dilemmas in physics education research. Paper presented at the American Educational Research Association Annual Meeting, Montreal, Canada.

Palmer, P. (2017). The courage to teach: Exploring the hidden landscape of a teacher's life (20th anniversary edition). Wiley Publishers.

Patrick, A., Martin, J. P., \& Borrego, M. (2022, in press). Critical research methods in STEM higher education: A state-of-the-art review. Journal of Women and Minorities in Science and Engineering.

Riley, D. (2017). Rigor/us: Building boundaries and disciplining diversity with standards of merit. Engineering Studies, 9(3), 249-265. DOI: 10.1080/19378629.2017.1408631

Secules, S., Gupta, A., Elby, A., \& Tanu, E. (2018). Supporting the narrative agency of a marginalized engineering student. Journal of Engineering Education, 107(2), 186-218. DOI: 10.1002/jee.20201

Secules, S., McCall, C., Mejia, J. A., Beebe, C., Masters, A. S. L., Sánchez-Peña, M., \& Svyantek, M. (2021). Positionality practices and dimensions of impact on equity research: A collaborative inquiry and call to the community. Journal of Engineering Education.

Singh, N. P. (2004). Black is a country: Race and the unfinished struggle for democracy. Cambridge, MA: Harvard University Press.

Slaton, A. E. (2010). Race, rigor, and selectivity in US engineering: The history of an occupational color line. Cambridge, MA: Harvard University Press.

Slaton, A. E., \& Pawley, A. L. (2018). The power and politics of engineering education research design: Saving the 'small n'. Engineering Studies, 10(2-3), 133-157. DOI: 10.1080/19378629.2018.1550785 
Smith, J. M., \& Lucena, J. C. (2016). How do I show them I'm more than a person who can lift heavy things?: The funds of knowledge of low income, first generation engineering students. Journal of Women and Minorities in Science and Engineering, 22(3), 199-221. DOI: 10.1615/JWomenMinorScienEng.2016015512

Sochacka, N. W., Walther, J., \& Pawley, A. L. (2018). Ethical validation: Reframing research ethics in engineering education research to improve research quality. Journal of Engineering Education, 107(3), 362-379.

Teti, M., \& Slaton, A.E. (2020). A picture is worth a 1000 lived experiences of illness: Photovoice as an emancipatory approach to public health and HIV. In Patterson \& Read (Eds.), The shapes of epidemics and global disease, Newcastle upon Tyne, UK: Cambridge Scholars Publishing.

Walther, J., Sochacka, N. W., \& Kellam, N. N. (2013). Quality in interpretive engineering education research: Reflections on an example study. Journal of Engineering Education, 102(4), 626-659. DOI: $10.1002 /$ jee. 20029

Valencia, R. R., \& Solórzano, D. G. (1997). The evolution of deficit thinking: Educational thought and practice. London: Falmer Press.

Zuberi, T., \& Bonilla-Silva, E. (Eds.). (2008). White logic, white methods: Racism and methodology. Lanham, MD: Rowman \& Littlefield Publishers.

Volume 28, Issue 1, 2022 
